\title{
Soft IP Design Framework Using Metaprogramming Techniques
}

\author{
Vytautas Štuikys, Robertas Damaševičius, Giedrius Ziberkas, and Giedrius \\ Majauskas \\ Kaunas University of Technology, Software Engineering Department \\ Studentu 50, 3031-Kaunas, Lithuania \\ \{stuik,damarobe,ziber,giedmaja\}@soften.ktu.lt
}

\begin{abstract}
We discuss the application of the metaprogramming techniques for soft IP design. Two metaprogramming paradigms are considered: the internal (using capabilities of the single language) and external (based on the usage of the target language and external metalanguage simultaneously) ones. The novelty of our approach is that we apply the concept of the multi-dimensional separation of concerns implemented via metaprogramming for the design of parameterized soft IPs for embedded systems in order to achieve higher flexibility, reusability and customizability.
\end{abstract}

Key words: soft IP, multi-dimensional separation of concerns, metaprogramming

\section{INTRODUCTION}

In recent years there has been a considerable progress in developing methods for automating design and co-design of embedded systems (ES) in both technological and methodological aspects [1,2]. However, the evergrowing complexity of ES combined with the requirement to decrease timeto-market is forcing hardware (HW) designers to apply concepts and methods previously used primarily in software (SW) design. The concept of soft IPs (aka virtual components - VCs) in HW design is a good example. The soft IPs are highly customizable components described either in HDLs such as VHDL and Verilog, or in programming languages like $\mathrm{C} / \mathrm{C}++$.

The design of soft IPs for ES is a complex task due to the following reasons. Having in mind the requirements for flexibility, scalability and 
customizability, a critical issue in the design of ES is their architecture. One way to reduce the architectural design time and costs is to reuse the preexisting soft IPs. However, smart products require various combinations of high performance, low cost and low power. The customization of IPs to fit application, if not pre-programmed, may require extensive design efforts.

Developers usually design general-purpose ES for reuse in numerous applications. Because the context of their usage is usually unknown, these designs often focus on the functionality issues only. On the other hand, the extreme specialization results in highly efficient design yet usable only in a single application. The design of highly reusable IP blocks doesn't solve the problem of adaptation for a particular context. The designer has to find the balance between the generalization of functionality and the specialization of performance characteristics.

The aim of this paper is (1) to formulate the role of the multidimensional separation of concerns $[3,4]$ in soft IP design. (2) To introduce the metaprogramming techniques and address their usage for designing customizable soft IPs. Our contribution and novelty is the analysis and application of the internal and external metaprogramming techniques based on the multi-dimensional separation of concerns (MDSoC).

The structure of the paper is as follows. We consider related works in Section 2. We explain the multi-dimensional separation of concerns and its role in Section 3. We consider the internal and external metaprogramming paradigms and their usage in soft IP design in Section 4. We present experiments, evaluation and conclusions in Sections 5,6 and 7, respectively.

\section{RELATED WORK}

In recent years there has been a great interest in dealing with the IP problems, as well as organized initiatives, such as VSIA. Lennard et al. [5] explicitly demonstrate the power of the separation of concerns in handling complex problems in system-level design. Seepold [6] focuses on the extension of the existing methodologies and generalisation to drive the development of $\mathbb{P}$ reuse and VCs. Vermeulen et al. [7] propose a systemlevel IP reuse methodology intended for soft and firm IP blocks. Designs are described in three layers of operations, which in combination allow structural and behavioural reuse.

The need for the customisation has been reported in [8]. The paper claims that even the most frequently reused components, although they implement a well-established standard, require a tiny customization to fit in the application. Chou et al. [9] use higher-level design abstractions that allow raising the level of abstraction of specifications above the low-level 
target-specific implementation thus allowing the designers to focus on global architectural and functionality decisions.

Faraboschi et al. [10] emphasize the role of scalability and customizability for ES. Rau et al. [2] distinguish most important features of ES architecture as follows. Specialization involves tailoring of reusable-butnot-efficient general-purpose ES to the application domain. Customization refers to a specialization of a similar available IP. Automation allows speeding up design using parameterized IP libraries and design frameworks.

Haase [11] proposes to use large libraries of highly parametric DesignObjects $^{T M}$ tailored in terms of customer requirements to support IP reuse. Givargis et al. [12] consider the parameterization of the entire HW architectures in terms of area, power, performance and functional requirements. Koegst et al. [13] focus on the specification of reuse-based designs targeting correct embedding and functional flexibility, and describe reuse-focused parameterization techniques.

New innovative paradigms of the MDSoC, multi-language specifications and multi-paradigm design are discussed in a number of papers. Jerraya $e t$ $a l$. [14] discuss the usage of multi-language specifications for system design. The design of a complex ES may require the combination of several specification languages for different aspects of implementation.

Ossher and Tarr $[3,4]$ introduce the concept of the MDSoC. Authors claim that major difficulties associated with SW reuse, component integration, system composition \& decomposition, and modifications of SW systems are due to a lack of the separation of concerns. They consider the separation of overlapping concerns, various dimensions (kinds) of concerns, and deal with interaction and integration of concerns. Although this field is still in the initial stage, there are reports of the benefits of the MDSoC. Murphy et al. [15] study the separation of concerns in OO systems. Kande and Strohmeier [16] apply the MDSoC in software architecture description, which allows considering systems from multiple perspectives.

\section{SEPARATION OF CONCERNS AND DESIGN}

\subsection{Motivation and role of the multi-dimensional separation of concerns}

The MDSoC has far-reaching and challenging objectives [3,4]: (1) to reduce SW complexity. (2) Improve comprehensibility. (3) Promote traceability throughout SW lifecycle. (4) Limit the impact of change, facilitating evolution and non-invasive adaptation and customization. (5) 
Facilitate reuse. (6) Simplify component integration.

There may be a variety of concerns used for describing and implementing a complex system. A particular concern may have multiple dimensions to be considered at different stages of the design process. The separation of concerns, however, is not enough for dealing with a complex system. Finally, concerns must be integrated. The environments and tools are actually integrated and specifically represented concerns, which address the prescribed objectives and requirements.

Concerns and their dimensions can be orthogonal (independent) and overlapping (dependable). It is comparatively easy to identify and integrate concepts for the orthogonal separation, e.g., the multi-language approach in SW/HW co-design $[1,14]$. The orthogonal separation can be introduced intuitively, presented implicitly and does not require the usage of an explicit model. In case of the overlapping concepts, we usually need to build a model in order to extract the benefits of the relationship as follows.

1. The concern "view" applied to an IP model has two dimensions: pure behavioral and pure structural. The functionality of the model can be revealed through the concern "process" which also has two dimensions: sequential and concurrent. For dealing with overlapping concerns, one need to have the relationship model like that proposed in [17] for transforming sequential VHDL models into concurrent ones for higher performance.

2. After analyzing VHDL and introducing the concern "role" of the VHDL constructs and their dimensions (lower-, higher-level construct), we can conceive metaprogramming in VHDL as will be explained in Section 4.

\subsection{Explicit MDSoC in design space}

The concept of the MDSoC is especially useful in the domain of ES design where a variety of requirements exist at different levels of abstraction. These requirements restrict the design space of soft IPs. We conceive the design space as a set of all feasible implementations of the domain model. The reuse library is an implementation of the design subspace [18]. The design space and concerns are the result of domain analysis.

The design space is multi-dimensional with multiple aspects of concern: data structure, behavior, performance, etc. Commonalties and variability of each aspect must be captured by suitable abstractions. These abstractions help to organize the multi-dimensionally related IPs into IP families, which in turn are composed of families of lower level IPs. Commonalties reflect the shared context that is invariant across the IP family, whereas the variations, which capture the distinguishing properties of IPs, have to be specified at a higher level of abstraction and represented via parameters. 
We represent the concerns explicitly via the generic parameter names, and the design space implicitly by the generic IPs. The design space is sliced by the generic parameters to meet the specific requirements (Figure 1, a).

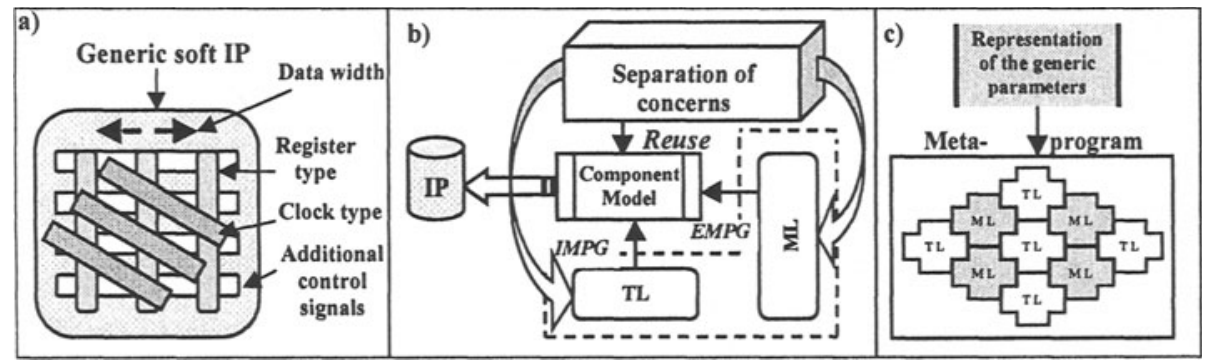

Figure 1. Our approach explained: (a) slices of concern in a generic register model; (b) the framework of IP design at the higher-level; and (c) composition of a metaprogram in EMPG

A component may be parameterized in terms of its functionality (specific functionality is selected from a family of the related one), size (data path width, RAM size, etc.), control (e.g. pipelining), performance characteristics (power, area, delay), synthesis tools and target technology. Slices do not exist in isolation; they can be interrelated differently. A metaprogram integrates slices into a single IP. The designer can instantiate a detailed component design, which is a specialization of a generic soft IP with respect to specified parameter values. We implement the concept of the MDSoC using the metaprogramming techniques as follows.

\section{METAPROGRAMMING AND SOFT IP DESIGN}

\subsection{Internal and external metaprogramming paradigms}

Metaprogramming (MPG) is the programming technique that enables a manipulation with other program structures. The higher-level metaprogram uses lower level constructs as data. The basis of MPG is a separation of the domain artifacts from the knowledge of how to customize and glue them together. This allows achieving program generalization and customization.

MPG can be implemented in several ways. At the abstraction level, we need to analyze the capabilities of the given target language (TL) and separate the concerns, which relate to implementing the basic functionality, from those, which allow expressing generic solutions and customized specifications. This separation may be accomplished, for example, implicitly using only the internal capabilities of the TL, or explicitly either introducing some extensions to the TL or using an external metalanguage (ML). 
At the implementation level, we need to build the architecture of the IP, to evaluate the properties of the soft IP model, and specify the required matching between properties using the appropriate abstractions. To achieve this, we separate the concerns at both the abstraction and IP model levels (Figure 1,b). We categorize two kinds of metaprogramming: the internal (IMPG) and external (EMPG) ones. The first one uses the internal capabilities of the TL, while the second uses TL and ML simultaneously.

The key features of IMPG are as follows. A designer: (1) works in one environment only (e.g., VHDL). (2) Implicitly separates the concerns within the environment. (3) Allocates a specific role for particular TL constructs: higher-level (e.g., if-generate, for-generate) for expressing the generalized functionality, and lower-level ones for expressing the domain functionality. (4) Manipulates with lower-level constructs using the higher-level ones.

The essence of the EMPG is as follows. A designer: (1) works in two environments. (2) Implements the separation of concerns explicitly, where $\mathrm{TL}$ is for basic domain functionality, and external ML is for customization, adaptation and generalization only. (3) Manipulates with TL using ML.

As the EMPG is still not matured enough, we explain the basic principles by analyzing the concrete languages (VHDL as a TL, and Open PROMOL [19] as a ML) as a case study for the EMPG for further discussion.

The concept of external functions is at the core of Open PROMOL, which has the following properties: (1) $\mathrm{ML}$ is an open set of external functions; it is TL-independent. (2) A function describes the manipulation with its arguments (parameters, TL code, or other functions). A variety of manipulations are supported in terms of structural programming. (3) External modules and nesting across the hierarchy of the external modules enhances flexibility of the hierarchical parameterization. (4) ML supports the conditional parameterization, i.e. the usage of a particular parameter dependent upon the pre-specified condition. (5) ML specification has a unified interface for customization of a given specification and interfacing with other specifications. (6) The result of processing of the PROMOL specification is an instance (in a TL) customized to the particular requirements of a designer.

We present some of these properties by a generic specification describing the majority vote function in VHDL with the introduced ML (Figure 2, a), and its instance (Figure 2, b). Note that the interface of a PROMOL specification is between a pair of ' $\$$ '. A generic specification is a composition of the HDL code, which describes features common for the entire IP family, and ML code, which specifies the slices of concerns represented by the generic parameters (Figure 1, c). 


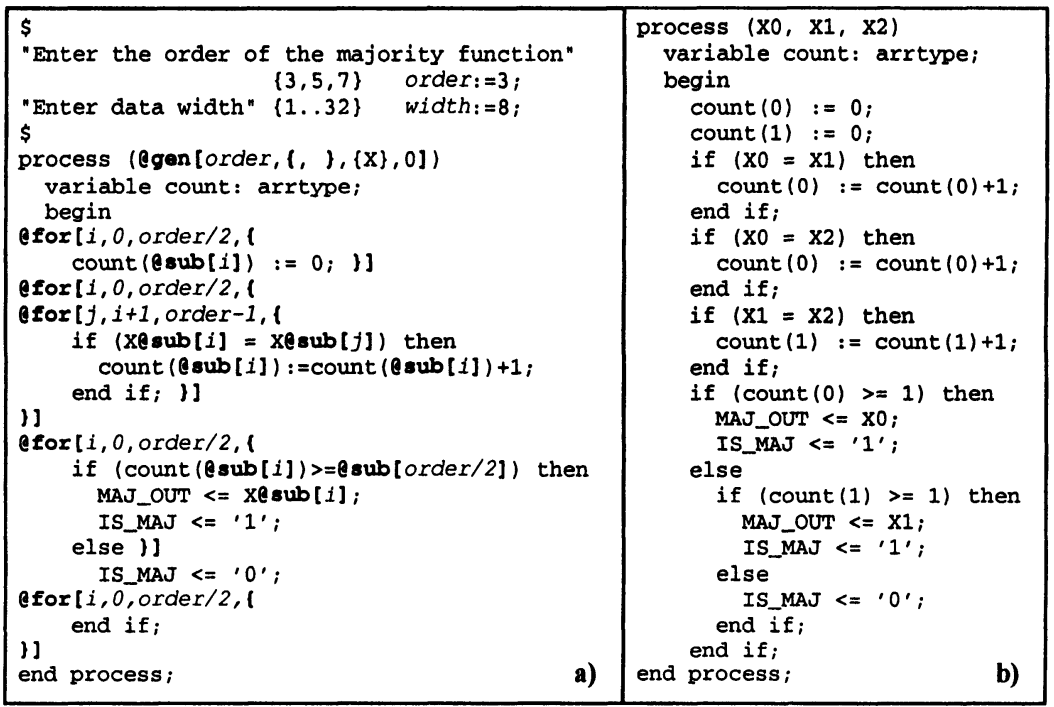

Figure 2. A metaprogram (fragment) implemented in Open PROMOL (a) and its instance (b)

\subsection{Comparison of IMPG and EMPG paradigms}

At the core of MPG are parameterization, customization and interfacing mechanisms. Although there are many similarities between the MPG techniques, both of them have some strengths and weaknesses (see Table 1).

Table 1. Comparison of parameterization and interfacing in MPG paradigms

\begin{tabular}{lll}
\hline \multicolumn{1}{c}{ MPG characteristics } & Internal MPG in VHDL & External MPG in ML \\
\hline Main mechanism & Generics, generate statements & External functions \\
Parameterization model & Hierarchical & Hierarchical \& conditional \\
Level of separation & Implicit (intuitive) & Explicit (external) \\
Customization interface & Package, generic map & Unified user-oriented \\
Instance naming & Automatic & Pre-programmed (manual) \\
\hline
\end{tabular}

The main strength of IMPG is the integration with existing tools and design methodologies. Only one design environment is required. When the number of parameters is small enough the method suits well for black-box reuse. However, this technique has some drawbacks. The method depends upon TL. Furthermore, components cannot be easily adapted for synthesis using different tools. Users often encounter the overgeneralization problem when they try to adapt the component manually.

The weaknesses of IMPG are often the strengths of EMPG (and vice versa). The usage of a TL-independent ML allows automatic (preprogrammed) adaptation to limitations of a particular synthesizer, and automatic documentation generation. The end-user has access to customized 
instances. An instance is much more readable than the generic component. This technique has some problems too. Firstly, it requires two design environments, thus the validation process is more complex. Secondly, the component name clashing must be prevented (manually or automatically).

\section{EXPERIMENTAL RESULTS}

We consider the following cases in our experiments: (1) Soft IP design from scratch for fine-grained and middle-grained components using IMPG. (2) The same as in the previous case, but using EMPG. (3) Customizations of the middle- and coarse-grained components using EMPG. (4) Wrapping third party IPs with tiny modifications using EMPG.

By tiny modifications we mean changing either the parameter value or library component in the retrieved IP in order to adapt it to the restrictions of our synthesis tools ${ }^{1}$. By wrapping we mean a family of the related instances, which differ in functional or/and technological characteristics, and are represented with EMPG technique as a single IP. Those characteristics and generic parameters for a wrapped generic multiplier IP are given in Table 2.

Table 2. Generic multiplier synthesis results

\begin{tabular}{|c|c|c|c|c|c|}
\hline \multicolumn{3}{|c|}{ Generic parameter values } & \multicolumn{3}{|c|}{ Synthesis results } \\
\hline $\begin{array}{c}\text { Multiplier } \\
\text { type }\end{array}$ & $\begin{array}{c}\text { Synthesis } \\
\text { tool }\end{array}$ & $\begin{array}{c}\text { Width, } \\
\text { bits }\end{array}$ & $\begin{array}{l}\text { No. of } \\
\text { cells }\end{array}$ & $\begin{array}{c}\text { Delay, } \\
\text { ns }\end{array}$ & Power \\
\hline \multirow{6}{*}{ Serial } & \multirow{3}{*}{ Cadence } & 16 & 441 & 27.84 & - \\
\hline & & 32 & 849 & 53.27 & - \\
\hline & & 64 & 1663 & 98.94 & - \\
\hline & \multirow{3}{*}{ Synopsys } & 16 & 324 & 7.32 & $3.7817 \mathrm{uW}$ \\
\hline & & 32 & 599 & 8.20 & $6.6184 \mathrm{uW}$ \\
\hline & & 64 & 1131 & 8.14 & $12.7298 \mathrm{uW}$ \\
\hline \multirow{6}{*}{$\begin{array}{l}\text { Mont- } \\
\text { gomery }\end{array}$} & \multirow{3}{*}{ Cadence } & 16 & 277 & 19.45 & - \\
\hline & & 32 & 540 & 29.91 & - \\
\hline & & 64 & 991 & 47.83 & - \\
\hline & \multirow{3}{*}{ Synopsys } & 16 & 355 & 2.80 & $9.5598 \mathrm{uW}$ \\
\hline & & 32 & 660 & 2.80 & $17.184 \mathrm{uW}$ \\
\hline & & 64 & 1237 & 2.80 & $32.4107 \mathrm{uW}$ \\
\hline \multirow[b]{2}{*}{ Booth } & \multirow[b]{2}{*}{ Synopsys } & 16 & 8 & 198.16 & $2.5410 \mathrm{~mW}$ \\
\hline & & 32 & 16 & 410.43 & $10.4788 \mathrm{~mW}$ \\
\hline
\end{tabular}

${ }^{1}$ We use Cadence (0.7 um) and Synopsys (1999.10) synthesis tools 


\section{EVALUATION AND DISCUSSION}

We have presented the framework for soft IP design methodology. The main focus has been given to the MDSoC and MPG techniques. The $\mathrm{MDSoC}$ can be considered as a pre-design analysis paradigm aiming at the identification, decomposition, encapsulation and integration of dimensions of concerns in order to improve the design characteristics. The MDSoC allows dealing with complex systems and raising the abstraction level of the design.

We demonstrate the usage of the MPG techniques, which are useful for customization, modification, wrapping, and generalization of IPs. Furthermore, the techniques allow generating soft IPs automatically and building more efficient and productive systems. We have also suggested the external MPG technique based on the usage of the external metalanguage (ML). The strength of the external MPG is its applicability to the different dimensions of TL models. Furthermore, by introducing an external ML we can extend the capabilities of MPG in soft IP design significantly.

On the other hand, the MDSoC and MPG techniques are still not matured enough, and a great deal of research must be done in the field of soft IP design. The suggested framework gives only preliminary results, but we believe in strength of the introduced techniques. The belief is also based on works of other authors who recognize and apply (at least implicitly) the concepts described explicitly in this paper.

\section{CONCLUSIONS AND FUTURE WORK}

The multi-dimensional separation of concerns is at the core in design of complex systems in general and soft IPs in particular. When introducing the separation of concerns we can understand better the design process and capture the essential domain artifacts to improve it. The internal and external metaprogramming techniques are only two cases at the abstraction level, which demonstrate the power of the separation of concerns. The external metaprogramming yields additional capabilities for customization and wrapping of soft IPs. The future work aims at extending dimensions at the soft IP model from customized IPs to the distributed customized soft IPs.

\section{REFERENCES}

[1] R. Ernst. Codesign of Embedded Systems: Status and Trends. IEEE Design and Test of Computers, April-June 1998, pages 45-54. 
[2] B.R. Rau, and M.S. Schlansker. Embedded Computer Architecture and Automation. IEEE Computer, April 2001, pages 75-83.

[3] H. Ossher, P. Tarr, W. Harrison, and S.M. Sutton, Jr. N Degrees of Separation: MultiDimensional Separation of Concerns. In Proceedings of the ICSE'99, 16-22 May 1999 Los Angeles, pages 107-119.

[4] H. Ossher, and P. Tarr. Multi-Dimensional Separation of Concerns and The Hyperspace Approach. In Software Architectures and Component Technology: The State of the Art in Software Development, ed. M. Aksit. Kluwer Academic Publishers, 2000.

[5] C. K. Lennard, P. Schaumont, and G. de Jong. Standards for System-Level Design: Practical Reality or Solution in Search of a Question? In Proceedings of the DATE 2000, 27-30 March 2000 Paris, pages 576-583.

[6] R. Seepold. Reuse of IP and Virtual Components. In Proceedings of the DATE'1999, 912 March 1999 Munich.

[7] A. Vermeulen, F. Catthoor, D. Verkest, and H. De Man. Formalized Three-Layer System-Level Reuse Model and Methodology for Embedded Data-Dominated Applications. In Proceedings of the DATE'2000, 27-30 March 2000 Paris, pages 92-98.

[8] J.F. Agaësse, and B. Laurent. Virtual Components Application and Customization. In Proceedings of the DATE'1999, 9-12 March 1999 Munich, pages 726-727.

[9] P. Chou, R. Ortega, K. Hines, K. Partridge, and G. Borriello. IPChinook: An Integrated IP-based Design Framework for Distributed Embedded Systems. In Proceedings of the $36^{\text {th }} A C M / I E E E$ on DAC, 21-25 June 1999 New Orleans, pages 44-49.

[10] P. Faraboschi, G. Brown, J.A. Fisher, G. Desoli, and F. Homewood. Lx: A Technology Platform for Customizable VLIW Embedded Processing. In Proceedings of the ISCA'00, 12-14 June 2000 Vancouver, pages 203-213.

[11] J. Haase. Design Methodology for IP Providers. In Proceedings of the DATE'1999, 912 March 1999 Munich, pages 728-732.

[12] T. Givargis, and F. Vahid. Parameterized System Design. In Proceedings of the CODES'2000, 3-5 May 2000 San Diego, pages 98-102.

[13] M. Koegst, P. Conradi, D. Garte, and M. Wahl. A Systematic Analysis of Reuse Strategies for Design of Electronic Circuits. In Proceedings of the DATE'98, 23-26 February 1998 Paris, pages 292-296.

[14] A.A. Jerraya, M. Romdhani, Ph. Le Marrec, F. Hessel, P. Coste, C. Valderrama, G.F. Marchioro, J.M. Daveau, and N.-E. Zergainoh. Multilanguage Specification for System Design and Codesign. In System Level Synthesis, NATO ASI 1998, eds. A.A. Jerraya, J. Mermet. Boston: Kluwer Academic Publishers, 1999.

[15] G.C. Murphy, A. Lai, R.J. Walker, and M.P. Robillard. Separating Features in Source Code: An Exploratory Study. In Proceedings of the $23^{\text {rd }}$ International Conference on Software Engineering, 12-19 May 2001 Toronto, pages 275-284.

[16] M. M. Kandé, and A. Strohmeier. On The Role of Multi-Dimensional Separation of Concerns in Software Architecture. In OPSLA'2000 Workshop on Advanced Separation of Concerns in Object-Oriented Systems, 15-19 October 2000 Minneapolis.

[17] A. Prihozhy, and M. Solomennik. Generating Concurrent Net Schedules from Sequential VHDL Models. In FDL '2001, 3-7 September 2001 Lyon.

[18] H.P. Peixoto, M.J. Jacome, A. Royo, and J.C. Lopez. The Design Space Layer: Supporting Early Design Space Exploration for Core-Based Designs. In Proceedings of the DATE'1999, 9-12 March 1999 Munich, pages 676-683.

[19] V. Stuikys, R. Damaševičius, and G. Ziberkas. Open PROMOL: An Experimental Language for Target Program Modification. In System-on-Chip Design Languages, eds. A. Mignotte, E. Villar, L.S. Spruiell. Kluwer Academic Publishers, 2002. 